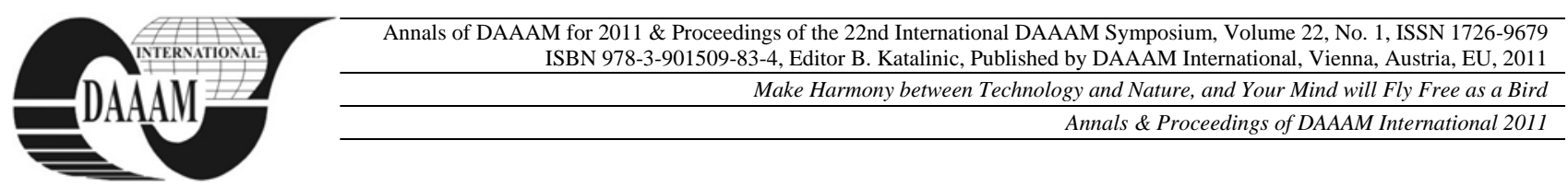

\title{
PORTABLE SURFACE PLASMON RESONANCE PHENOMENON (SPR)-BASED BIOSENSORS
}

\section{GUTT, S[onia]; GUTT, G[heorghe]; GUTT, A[ndrei]; POROCH - SERITAN, M[aria]; MIRONEASA, S[ilvia] \& ROSU, A[lice] I[uliana]}

\begin{abstract}
Two new solutions of developing highly performing portable surface plasmon resonance phenomenon-based (SPR) biosensors with applications especially in immune detection are described. The first biosensor is laboratory equipment having an optoelectronic structure with information transmission by optical fiber and uses an interferometer structure of Michelson type to measure wave length, leading to increased resolution and measurement accuracy when determining the layer mass or thickness by the SPR method. The second biosensor is portable equipment for in situ determinations, based also on optical fibre structure and uses a Diode-Array detector to quantify the wavelength of the radiation refracted.
\end{abstract}

Key words: optical fibre, diode-array, interferometer, evanescent field

\section{INTRODUCTION}

Surface plasmon resonance (SPR) is a new method of measuring mass variations reaching up to the level of $10^{-15}$ grams (femtograms), thus rendering SPR the most notable method of mass balance known so far (Isfort, 2005). The classical technique SPR uses monochromatic radiation of laser type which is perpendicularly sent on the face of equilateral glass prism, by crossing it, and gets on the opposite face where a thin golden or silver layer is deposited on, thus generating total reflection of radiation with an angle equal to the incidence one.

The photons of laser radiation falling on the metallic golden or silver coating interfere with the free ions of these metals and form a cloud of free ions called "surface plasmon resonance". If there is resonance between the free ions from plasmon structure and photons of the monochromatic irradiation source, then the part of energy spent to maintain resonance is extracted from the radiation reflected by the golden or silver layer, therefore some energy loss occurs in the reflection spectrum which is a variation of the radiation reflection angle which goes out through the third face of glass prism against the value of angle specific to the lack of plasmon resonance(Achilleos et. al., 2009)

This variation is under the form of a measurable refraction angle which value depends on the intensity of surface plasmon resonance. Plasmon resonance shows itself energetically also on the exterior side of the metallic golden or silver layer through a so called "evanescent field" (Gang Xu et. al., 2008) which intensity reacts extremely sensitively to any mass change occurring on this exterior side. This phenomenon is the basis of detecting the change of mass or layer thickness on this surface. For real applications a specific receiver is immobilized on the sensor surface which is supposed to interact with a potential addend ligature to be determined. If interaction occurs between the receiver and the species focused on, then the mass loading degree of metal layer increases proportionally and the level of surface plasmon resonance increases also proportionally, the refraction angle corresponding to this level is measured by hand, either by an optical goniometer or automatically (Gutt et al., 2008), (Gutt et al., 2009a, 2009b, 2009c) by means of a
Diode-Array detector placed on the prism face where the reflected and refracted radiation gets out from.

\section{EXPERIMENTAL}

The authors have presented two conceptual and constructive solutions for performing biosensors based on SPR.

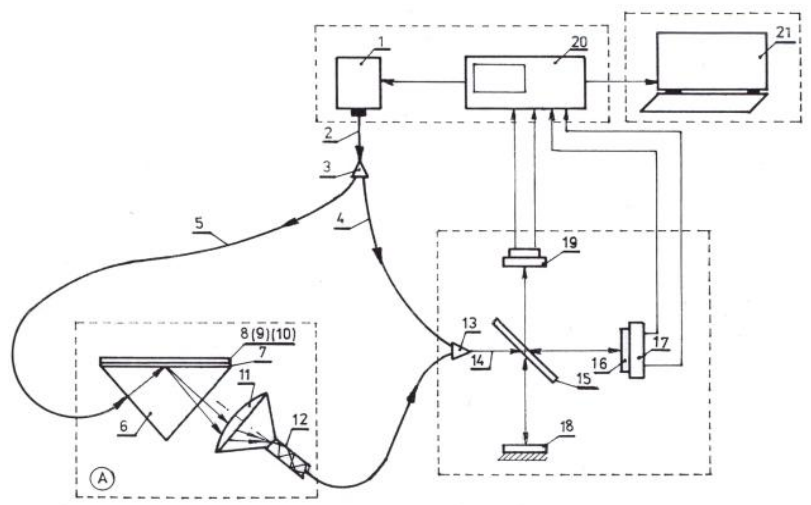

Fig. 1. Basic diagram of interferometer laboratory biosensor based on surface plasmon resonance phenomenon: 1 - radiation source of laser diode type, 2 - optical fibre, 3 - optical divider, 4, 5 - optical fibres, 6 - optical prism, 7 - thin golden or silver deposit, 8 - layer of adherence, 9 - active substance, 10 chemical or biological structure aimed at, 11 - focusing lens, 12 - optical fibre of transmission, 13 - optical adder, 14 -optical fibre, 15 - semi transparent mirror, 16 - mobile mirror with total reflection, 17 - linear piezoelectric motor, 18 - fixed mirror with total reflection, 19 - photo electric detector, 20 - central electronic unit, 22 - computing unit

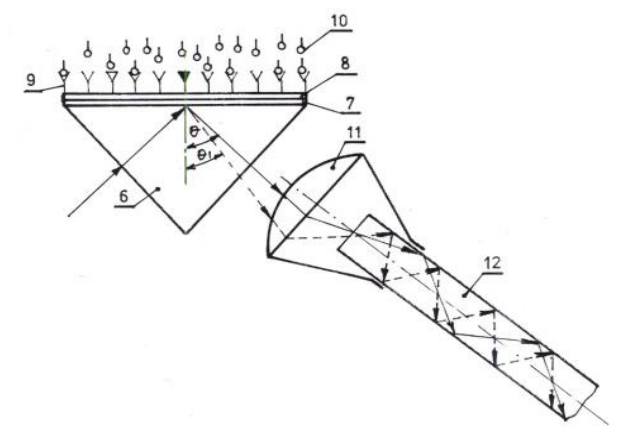

Fig. 2. Detail A of basic diagram on radiation propagation into a structure of surface plasmon resonance and connecting optical fibre with interferometer. Basic diagram of interferometer laboratory biosensor based on surface plasmon resonance phenomenon. 6 - optical prism, 7 - thin golden or silver deposit, 8 - layer of adherence, 9 - active substance, 10 - chemical or biological structure aimed at, 11 - focusing lens, 12 - optical fibre of transmission 
In this sense, figure 1 shows a laboratory biosensor which wavelength, resulted from the refraction made by the evanescent field, is measured by interferometer.

A monochromatic radiation emitted by a laser diode, by means of an optical fibre, is divided into two parallel beams, one of the optical (reference) beams goes, through optical fibre, round the measuring structure of the refraction angle by SPR technique and reaches an optical adder.

The other (work) beam is guided by means of another optical fibre towards the system of surface plasmon resonance where it falls perpendicularly on the transparent face of an optical prism, is reflected from a golden coating deposited on thin layer on another face of the prism and leaves it through its third transparent face, reaching an optical convergent lens which focuses radiation on an optical fibre which on its turn meets, by means of an adder, the optical fibre transporting the reference beam, the two light signals falling, after they have merged, on a semi transparent mirror of an interferometer of Michelson type.

The second biosensor made by our team, figure 3 , is of portable type and also based on a monochromatic radiation source of laser diode type which transmits light radiation through a flexible optical fibre to an optical coupler, the latter one permitting fast and by turn connection of some interchangeable SPR wells, under the form of cylindrical rods with stainless coat, inside which there is polymer filling wherein a pack of twelve optical thin fibres (diameter of almost 100 of $\mu \mathrm{m}$ ) is circularly distributed around a central optical fibre of larger diameter (hundreds of $\mu \mathrm{m}$ ). At the end opposite to fixing in the optical coupler, the probe has a plane circular surface on which a thin pure golden layer is deposited, and on this one, a thin prepared laboratory layer is deposited too, containing biologically active components that are to interact with the species focused on. In the case when biosensor is used as immunosensor, a gel is being deposited on the golden layer which contains antibodies specific to an antigen aimed at in the

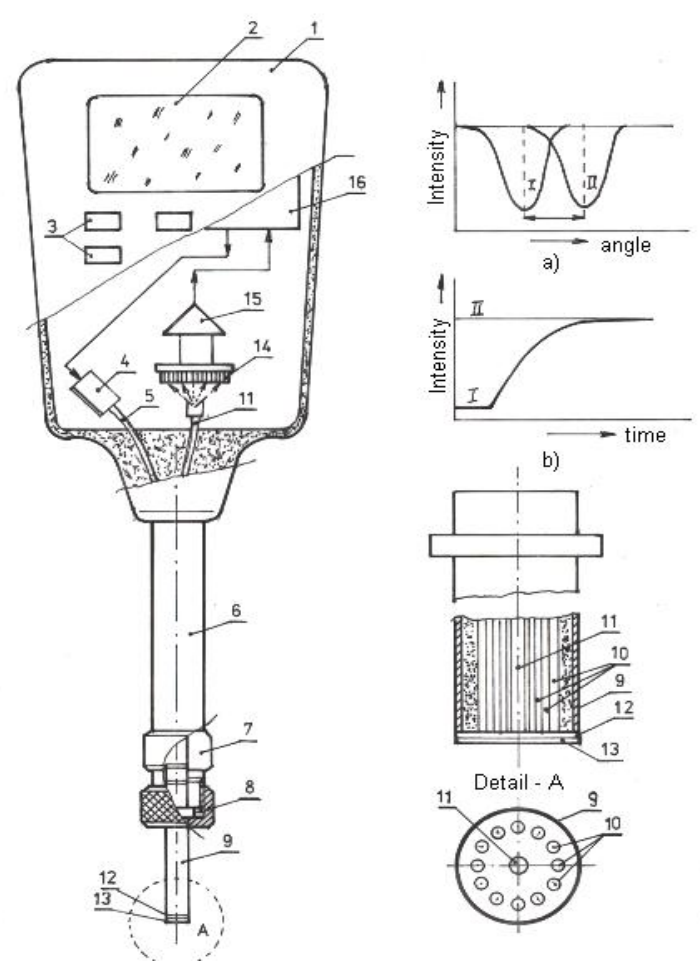

Fig. 3. Basic diagram of portable biosensor: 1 - body, 2 - alpha numerical display, 3 - setting buttons, 4 - laser diode, 5 - optical fibre, 6 - connecting body, 7 - optical divider head, 8 - screwing nut, 9 - changeable well with other wells with biological support prepared, 10 - optical fibre of irradiation, 11 - central optical fibre, 12 - thin golden layer, 13 - thin layer of biologically active material, 14 - Diode-Array detector, 15 electronic amplifier, 16 - microprocessor

biological species researched

As a result of light irradiation of the thin golden layer, plasmon resonance excitation from the twelve optical fibres takes place, and the system answer, expressed by a certain value of the refraction angle, is transmitted through the central optical fibre to an optoelectronic detector of photodiode array type (Diode-Array), which consists of almost 4.000 of photodiodes, fact that provides the system with special measuring resolution.

\section{RESULTS AND DISCUSSION}

Two performing biosensors based on SPR phenomenon are made. The laboratory biosensor has high performances as it uses a Michelson interferometer and a Fourier transform to measure the refraction angle.

By this solution the measuring resolution is half the value of wavelength used in irradiation $(\lambda / 2)$, level which is almost 10 $\div 10^{2}$ times better than that obtained from classical goniometric measurement of refraction angle, which has as direct effect the increase of measuring accuracy of mass and layer thickness respectively of the species focused on.

The portable biosensor has also high performances as it uses a Diode - Array detector to measure refraction angle, solution that gives a measuring resolution of almost $10^{2}$ times higher than the classical method with optical goniometer. But mention should be made that in the case of this interferometer, sensitivity is lower due to the optical information division in high number of receiving diodes.

\section{CONCLUSION}

Plasmon resonance-based biosensors are at present the only highly performing and relatively cheap means, having detection limits for mass variations that may reach the level of $10^{-15}$ grams.

To ensure high measuring resolution and accuracy is the most important factor of this type of biosensor.

Other requirements may be added as well, such as automatic measurement of the refraction angle caused by SPR, the possibility of pursuing fast kinetics processes, using wells of fast interchangeable active layer, performing in situ analyses, ratio signal/reduced noise.

\section{REFERENCES}

Achilleos, C.; Tailhardat, M.; Courtellemont, P.; Le Varlet, B. (2009). Dupont Investigation of surface plasmon resonance biosensor for skin sensitizers studies, $D$. Toxicology in Vitro, 23, 308-318, ISSN: 0887-2333

Gang, Xu; Chun-Ming, H.; Masato, T.; Ping, J.; Hua Chen, L.. (2008), Tunable optical properties of nano-Au on vanadium dioxide, Optics Communications, 282, Elsevier, 896-902, ISSN: 0030-4018

Gutt, S.; Gutt, G.; Gutt, A.; (2009a), Biosensor for measuring changes in mass and thick layer, Proposal for invention. A00847,(2009), OSIM Bucuresti,

Gutt, S.; Gutt, G.; Gutt, A.. (2009c). Miniature Biosensors , Proposal for invention A-00854,(2009), OSIM Bucuresti,

Gutt, S.; Gutt, G.; Gutt, A.. (2008). Biosensor, Patent RO125798-a2/2010, OSIM Bucuresti

***Isfort, G.D.; (2005). Oberflächenplasmonenresonanz von passivierten Silberschichten: Polarisation und Anisotropie der Lichtemission bei Kopplung an fluoreszierende und Raman-streuende Moleküle, Disertation, Düsseldorf 